\title{
TEXTURE, PIEZOELECTRICITY AND FERROELECTRICITY
}

\author{
L. Fuentes ${ }^{1}$ and O. Raymond ${ }^{2}$ \\ 'Instituto de Cibernética, Matemática y Física, Habana, Cuba \\ ${ }^{2}$ Instituto Superior Pedagógico E. J. Varona, Habana, Cuba
}

(Received 9 March 1995)

\begin{abstract}
A Quantitative Texture Analysis approach to polycrystal piezoelectric and ferroelectric phenomena is given. Monocrystal longitudinal piezoelectric moduli are expanded in Bunge's symmetry- adapted functional bases. Suitable expansion coefficients are given. Orientation Distribution Function based algorithms for polycrystal piezo-moduli prediction are presented. Significant odd-order expansion terms are calculated and their relation to ghost phenomena is commented. Polycrystal ferroelectricity is characterized. Quantitative describers associated to crystallographic and electric orientation distributions are presented and related. Their evolution during heat and poling processes is discussed. Two computersimulated examples are analyzed: (a) Texture-modulated longitudinal piezo-modulus is calculated for an ideal quartz single-component texture. (b) Barium titanate fibre texture transformation during a hypothetical technological process is investigated.
\end{abstract}

KEY WORDS: Piezoelectricity, ferroelectricity, non-centrosymmetric crystals, direct ghost correction

\section{INTRODUCTION}

Piezoelectric and ferroelectric materials are technologically relevant. Their properties are characterized in several important works (see, for example, Berlincourt et al, 1964; IEEE Standards on Piezoelectricity, 1987; Mitsui and Nakamura, 1989; Gallego Juárez, 1989; Ikeda, 1990; Zgonik et al, 1994). From the physical point of view, they are interesting, particularly in relation to the role of symmetry in the piezoelectric phenomenon. As to the connection with texture analysis, the subject shows a peculiar link to so-called ghost phenomena, so it deserves detailed consideration. Piezoelectric pole figures of a vien quartz sample were reported by Ghomshei et al (1988).

We have not found a systematic texture-oriented treatment of this subject in the literature. The purpose of the present work is, therefore, to characterize piezoelectric and ferroelectric phenomena from the Mathematical Texture Analysis point of view. Textures are described by means of orientation distribution functions (ODFs), following Bunge's (1982) symmetry-adapted spherical harmonics expansions. Electric variables are also described under these algorithms. Formulae are given for prediction of piezoelectric parameters in polycrystal samples with known orientation distributions. Two hypothetical computer-generated examples are discussed.

Some basic statements of this article are electric variants of Bunge's (1989) ideas about magnetic textures. 


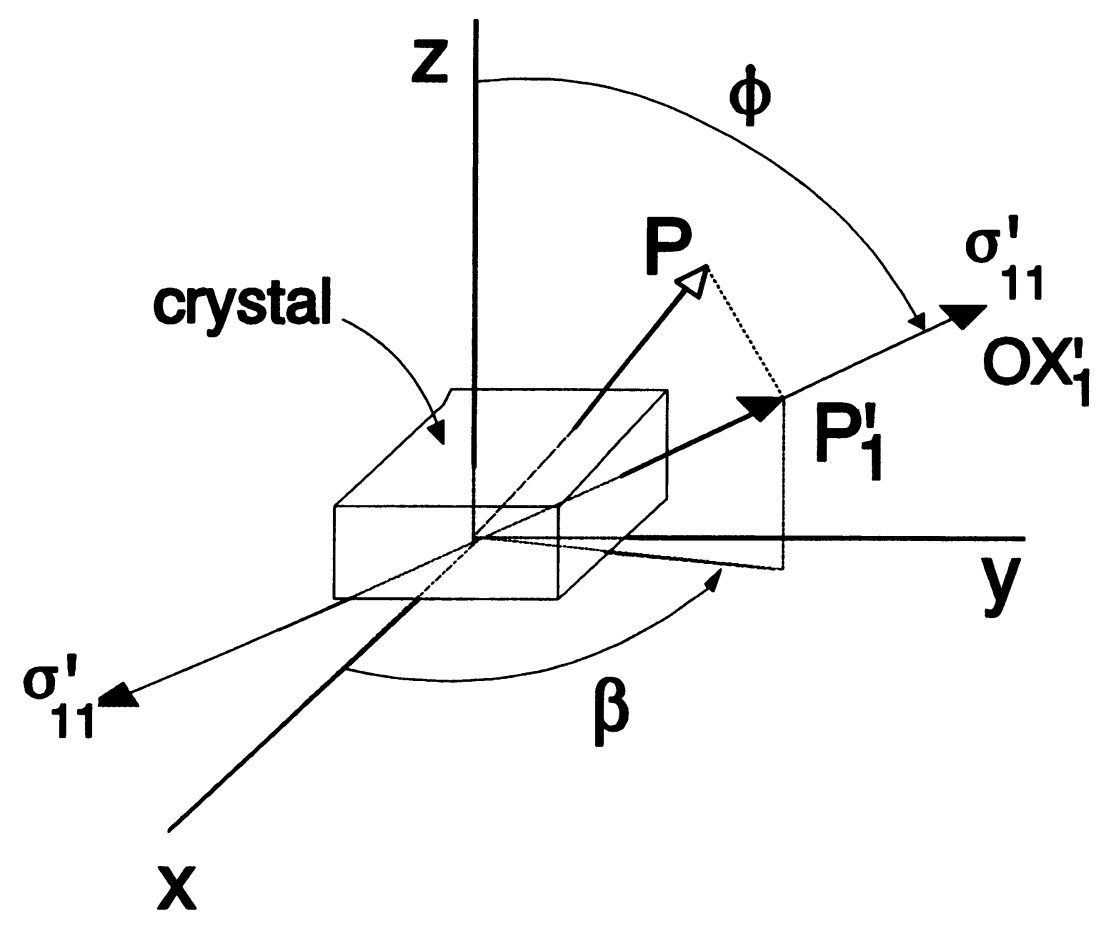

Figure 1 Definition of longitudinal piezoelectric modulus.

\section{MONOCRYSTAL PIEZOELECTRICITY}

Piezoelectricity is an exclusive property of non-centrosymmetrtic crystals. This fact follows from Neumann's Principle (see, for example, the International Tables of Crystallography, 1983).

The magnitude that usually characterizes the piezoelectric response of a given crystal is its piezoelectric tensor $d_{i j k}$. It is a third-rank tensor, relating electric polarization $P_{i}$ and stress $\sigma_{j k}$ according to:

$$
P_{j}=\sum_{j} \sum_{k} d_{i j k} \sigma_{j k}
$$

Nye's (1957) classic book contains a detailed presentation of this tensor's properties. It is common practice to write it as a $3 \times 6$ matrix, with crystal point-group symmetry simplications.

A scalar description of the piezoelectric effect is given by the so-called "longitudinal piezoelectric surface". We give its definition with the help of Figure 1.

Consider an arbitrary direction $h=O X_{1}^{\prime}=(\Phi, \beta)$ in the observed crystal. Let a pure traction stress $\sigma_{11}^{\prime}$ be applied along $O X_{1}^{\prime}$. The resulting polarization vector $P$ will not point (as a rule) parallel to $O X_{1}^{\prime}$. Project $P$ over $O X_{1}^{\prime}$ and denote the obtained 
projection by $P_{1}^{\prime}$. The longitudinal piezoelectric modulus $d(h)=d_{111}^{\prime}(h)$ is then defined by:

$$
P_{1}^{\prime}=d(h) \sigma_{11}^{\prime}
$$

Now let $O X_{1}^{\prime}$ scan all directions in space and plot the surface determined by the ending points of all vectors $d$ having orientation $O X_{1}^{\prime}$ and moduli $d(h)$. This is the "longitudinal piezoelectric surface".

To calculate $d(h)$ the following equation holds:

$$
d(h)=\sum_{i} \sum_{j} \sum_{k} a_{1 i} a_{1 j} a_{1 \mathrm{k}} d_{i j k}
$$

$a_{1 i}, a_{1 j}, a_{1 k}$ are the direction cosines of $h$ relative to the cartesian axes $O X_{1}, O X_{2}$, $O X_{3}$.

As implied by the symmetry properties of the tensor $d_{i j k}$, there are specific expressions for $d(h)$ associated to each crystallographic (non-centric) point group. Two important cases deserve attention. These are the trigonal $D_{3}=32$ and tetragonal $C_{4 v}=4 \mathrm{~mm}$ point groups. They describe, respectively, the macroscopic symmetries of quartz and of ferroelectric perovskites (barium titanate, some "PZT" ceramics). The associated equations are:

$\rightarrow 32$ point group:

$$
d=d_{11} \sin ^{3} \phi \cos 3 \beta
$$

$\rightarrow 4 \mathrm{~mm}$ point group:

$$
d=\left(d_{33}-d_{15}-d_{31}\right) \cos ^{3} \phi+\left(d_{15}+d_{31}\right) \cos \phi
$$

$d_{i j}$ are expressed in matrix notation. Experimental values, for quartz and barium titanate, are:

$\rightarrow$ quartz:

$d_{11}=2,31 ; \quad d_{14}=0.727 \quad\left(10^{-12} \mathrm{C} \mathrm{N}^{-1}\right)$

$\rightarrow$ barium titanate:

$d_{33}=85.5 ; \quad d_{31}=-34.5 ; \quad d_{15}=.392 \quad\left(10^{-12} \mathrm{CN}^{-1}\right)$

An alternative treatment for $d(h)$ is given by its symmetry-adapted spherical harmonics $\left(k_{I}^{\mu}(h)\right)$ expansion. In this framework:

$$
d(h)=\sum_{l=1(2)}^{3} \sum_{\mu=1}^{M(1)} e_{l}^{\mu} k_{l}^{\mu}(h)
$$

The upper limit of the sum over $\mu$ depends on 1 according to point-group determined rules. 
The coefficients $e_{1}^{\mu}$ are obtained on the basis of orthogonality relations associated to $k_{I}^{\mu}(h)$ :

$$
e_{1}^{\mu}=\oint d(h) k_{1}^{\mu}(h) d h
$$

A suitable set of real triclinic spherical harmonics $k_{l}^{\mu}(h)(l=1,3)$ is suggested in Appendix I. This collection contains complete functional bases $k_{I}^{\mu}(h)$ for piezoelectric moduli expansions in all cases associated to crystals of lower-than-cubic symmetry.

Some crystals, like barium titanate, show axially symmetric piezoelectricity (see equation 5). For such cases, equations (6) and (7) may be written, respectively:

$$
\begin{gathered}
d(\phi)=e_{1} P_{1}(\phi)+e_{3} P_{3}(\phi) \\
e_{1}=\int_{0}^{\pi} d(\phi) P_{1}(\phi) d \phi \quad l=1,3
\end{gathered}
$$

$P_{1}$ are Legendre polynomials (see Appendix I).

For 32 and $4 \mathrm{~mm}$ point groups the expressions of $d(h)$ as symmetry-adapted spherical harmonics expansions are:

$\rightarrow 32$ group:

$$
\begin{aligned}
& d(h)=e_{3}^{1} k_{3}^{1}(h) \\
& e_{3}^{1}=4 \sqrt{\frac{2 \pi}{35}} d_{11}
\end{aligned}
$$

with trigonal $k_{3}^{1}(h)$ being equal to triclinic $K_{3}^{7}(h)$.

$\rightarrow$ 4mm group:

$$
\begin{gathered}
d(h)=e_{1}^{1} k_{1}^{1}(h)+e_{3}^{1} k_{3}^{1}(h)=e_{1} P_{1}(\phi)+e_{3} P_{3}(\phi) \\
e_{1}^{1}=\frac{2}{5} \sqrt{3 \pi}\left[d_{33}+\frac{2}{3}\left(d_{15}+d_{31}\right)\right]=\sqrt{2 \pi e_{1}} \\
e_{3}^{1}=\frac{4}{5} \sqrt{\frac{\pi}{7}}\left(d_{33}-d_{15}-d_{31}\right)=\sqrt{2 \pi e_{3}}
\end{gathered}
$$

with tetragonal $k_{1}^{1}(h)$ and $k_{3}^{1}(h)$ being given, respectively, by triclinic $K_{1}^{2}(h)$ and $K_{3}^{4}(h)$.

\section{TEXTURE CHARACTERIZATION}

We shortly present the Orientation Distribution Function (ODF) and other important magnitudes of Mathematical Texture Analysis. For detailed explanations, see Bunge (1982). 
The ODF, $f(g)$, expresses the volume fraction $d V$ of crystallites in the neighborhood $d g$ of the orientation $g=\left(\varphi_{1}, \phi, \varphi_{2}\right)$ :

$$
\frac{d V}{V}=f\left(\varphi_{1}, \varphi, \varphi_{2}\right) d g
$$

$\left(\varphi_{1}, \phi, \varphi_{2}\right)$ are the Euler angles, describing the orientation of crystal system $K_{B}$ with respect to sample system $K_{A}$. The domain of $f(g)$ is:

$$
\Re_{3}: \quad 0 \leq \varphi_{1}, \varphi_{2} \leq 2 \pi ; \quad 0 \leq \phi \leq \pi
$$

The volume differential in orientation (Euler) space is given by:

$$
d g=\frac{1}{8 \pi^{2}} \sin \phi d \phi d \varphi_{1} d \varphi_{2}
$$

The ODF satisfies the normalization condition:

$$
\oint f(g) d g=1
$$

Sample and crystallite symmetries play important roles in texture phenomena. The ODF is invariant under proper rotations (inversion not included) that belong to the respective point groups. Let the proper rotations from sample symmetry be denoted by $g_{A_{i}}$ $\left(i=1, \ldots, N_{A}\right)$ and those from crystal symmetry by $g_{B_{j}}\left(j=1, \ldots, N_{B}\right)$. Then the following relation holds:

$$
f(g)=f\left(g_{A_{i}} \cdot g \cdot g_{B_{j}}\right)
$$

By symmetry, the definition domain of $f(g)$ in Euler space is $N_{A} N_{B}$ times reduced.

Three-dimensional spherical harmonics expansion of $f(g)$ is necessary in the present study. The fundamental equation in this treatment is:

$$
f\left(\varphi_{1}, \phi, \varphi_{2}\right)=\sum_{l=0}^{\infty} \sum_{\mu} \sum_{v} C_{1}^{\mu v} T_{1}^{\mu v}\left(\varphi_{1}, \phi, \varphi_{2}\right)
$$

$T_{I}^{\mu v}(g)$ represent the mentioned harmonics and $C_{I}^{\mu v}$ are expansion coefficients. Upper limits for $\mu$ and $v$ depend on crystal and sample symmetries. Orthogonality conditions for $f(g)$ allow the calculation of $C_{I}^{\mu v}$ on the basis of the knowledge of $f(g)$ :

$$
C_{I}^{\mu v}=(2 l+1) \int f(g) T_{I}^{\mu v} d g
$$

Explicit algorithms for low-symmetry odd-order real generalized harmonics are to be published (Raymond et al.).

Other quantitative descriptions of crystallographic textures are those given by direct and inverse pole figures (CDPF, CIPF). Here they will be denoted, respectively, $Q_{h}^{c}(y)$ and $R_{y}^{c}(h)$. Pole figures are two-dimensional projections of the three-dimensional ODF. 
Frequently measured CDPF and CIPF are those corresponding to $h=[0,0,1]=z_{B}$ for $Q_{h}^{c}(y)$ and $\mathrm{y}=z_{A}$ for $R_{y}^{c}(h)$ :

$$
\begin{aligned}
& Q_{z_{B}}^{c}(y)=\frac{d V\left(y \|_{z_{B}}\right) / V}{d y}=\frac{1}{2 \pi_{y} \|_{z_{B}}} \int_{f}(g) d \psi \\
& R_{z_{A}}^{\mathrm{c}}(h)=\frac{d V\left(y \|_{z_{A}}\right) / V}{d y}=\frac{1}{2 \pi_{h} \|_{z_{A}}} \int f(g) d \psi
\end{aligned}
$$

If the orientation distribution is symmetric with respect to rotations about a given axis, then we have a fibre texture. in this case the CIPF of the symmetry axis plays the role of the ODF. For this situation, relations (20) and (21) are substituted by:

$$
\begin{aligned}
& R(h)=\sum_{l} \sum_{\mu} C_{l}^{\mu} k_{l}^{\mu}(h) \\
& C_{l}^{\mu}=\oint_{R(h) k_{l}^{\mu}(h) d h}
\end{aligned}
$$

\section{POLYCRYSTAL PIEZOELECTRICITY}

Consider a textured polycrystalline sample, characterized by $f(g)$. By application of the so-called Hill approximation, it is possible to represent the average piezoelectric effect by the simple mean of the longitudinal piezoelectric modulus $\langle d\rangle$. The mean approximation is equivalent to the assumption of equal stresses for all crystallites in the sample. Using Bunge's method for mean surface representations, we write:

$$
<d>=\sum_{l=1}^{3} \sum_{v} \xi_{l}^{v} k_{l}^{v}(y)
$$

where $k_{l}^{v}(h)$ are spherical harmonics associated to sample symmetry and the coefficients $\xi_{1}$ are related to texture by:

$$
\xi_{I}^{v}=\frac{1}{2 l+1} \sum_{\mu} C_{I}^{\mu v} e_{I}^{\mu}
$$

Observe the important point that $1=1,3$. The needed $C^{\mu v}{ }_{1}$ are not detectatble by normal diffraction. The prediction of $\langle d\rangle$ demands precisely of the generally unknown "odd" part of $f(g)$; the component whose knowledge would avoid "ghost phenomena". Standing from another point of view, the measurement of $\langle d\rangle$ as a function of direction $y$ in the sample, plus the use of equations (26) - (27) would lead to gross corrections (up to $1=3$ ) for ghost phenomena.

If the texture shows axial symmetry (fibre texture), equations (26) and (27) are simplified to:

$$
<d>=\xi_{1} P_{1}(\phi)+\xi_{3} P_{3}(\phi)
$$




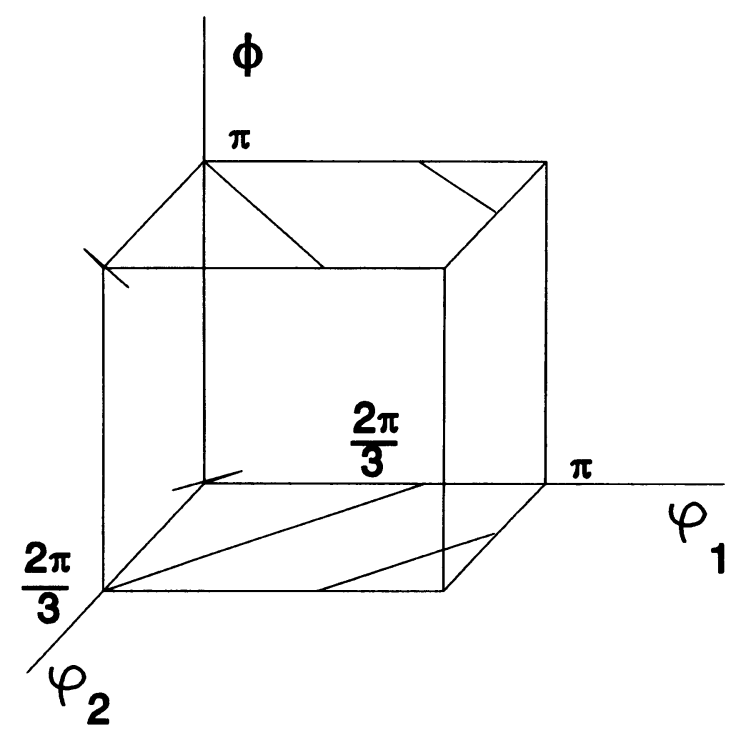

Figure 2 Definition domain and multiplicity for quartz model texture.

$$
\xi_{1}=\frac{1}{4 \pi} \sqrt{\frac{2}{2 l+1}} \sum_{\mu} e_{1}^{\mu} C_{1}^{\mu v} \quad l=1,3
$$

The special case of fibre texture (eqs. 28 and 29), combined with crystal axial piezoelectricity (eqs. 8 and 9), will be characterized by means of an example, below.

\section{MODEL TEXTURES IN QUARTZ}

We now present the study of a hypothetical case, associated to computer-generated textures. The system is made up of quartz crystallites, with only one enantiomorphic type in the sample. The orientation distribution consists of a collection of gaussian components, $f_{j}(g)$, centered around the Euler space origin $g_{1}^{*}=(0,0,0)$ and around the equivalent points induced by symmetry, $g_{j}^{*}=g_{A_{i}} \cdot g_{1}^{*} \cdot B_{k}$. The definition equation for $f(g)$ is:

$$
f(g)=f_{0} \sum_{j} \mathrm{e}^{-\left(\Omega_{j} / \Omega_{0}\right)^{2}}
$$

$\Omega_{j}$ is the angular distance between the orientation $g$ and the considered component maximum $g_{j}^{*}$. The width $\Omega_{0}$ and amplitude $f_{0}$ are common to all components. $f_{0}$ is such that the normalization condition (18) holds.

The considered system shows triclinic sample symmetry, point group $C_{1}=1\left(N_{A}\right.$ $=1$ ) and trigonal crystal symmetry, point group $\mathrm{D}_{3}=32\left(\mathrm{~N}_{\mathrm{B}}=6\right)$. Figure 2 represents the minimum region in Euler space needed for unique definition of $f(g)$. The maximum of $f_{0}$ reproduces itself over the shown lines. 


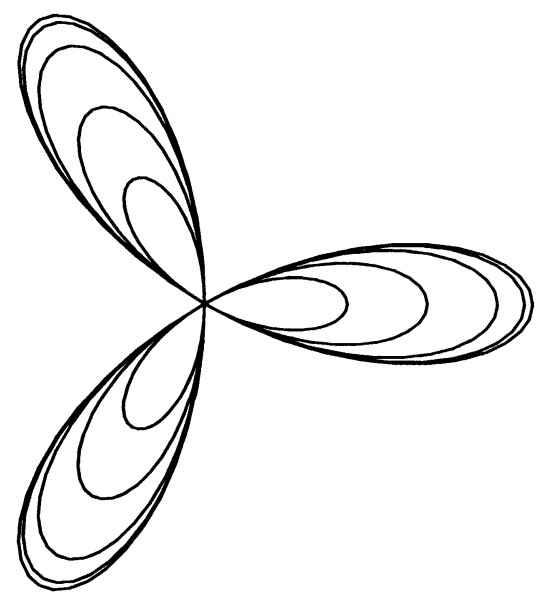

$\begin{array}{cc}\Omega_{0}\left(^{0}\right) & d(\text { polycr }) / d(\text { monocr }) \\ 0 & 1.000 \\ 5 & 0.975 \\ 10 & 0.894 \\ 20 & 0.681 \\ 30 & 0.437\end{array}$

Figure 3 Quartz polycrystals. Longitudinal piezoelectrric surface $(\Phi=\pi / 2)$

By symmetry, the calculation of $C_{I}^{\mu v}$ for the considered system reduces to:

$$
C_{I}^{\mu v}=\frac{2 l+1}{S} \oint \mathrm{e}^{-\left(\Omega_{1} / \Omega_{0}\right)^{2}} T_{1}^{\mu v} d g \quad(l=3 ; \mu=1 ; v=1, \ldots, 7)
$$

with

$$
S=\oint e^{-\left(\Omega_{1} / \Omega_{0}\right)^{2}} d g
$$

For the component at origin, the angular distance $\Omega_{1}$ is given by (Helming and Matthies, 1984):

$$
\cos \Omega_{1}=\frac{1}{2}\left[(1+\cos \phi) \cos \left(\varphi_{1}+\varphi_{2}\right)+\cos \phi-1\right]
$$

Appendix II shows triclinic-trigonal tridimensional spherical harmonics, for $1=3$. These functions, together with bidimensional harmonics of Appendix I, conform a complete functional basis for the characterization of the considered polycrystal piezoelectricity problem.

Case $\Omega_{0} \rightarrow 0$. In this situation, the ODF $f(g)$ tends to a collection of Dirac delta 
functions $\delta\left(g-g_{j}^{*}\right)$. Physically the problem is that of a polycrystal that tends to a monocrystal. $C_{3}^{1 v}(v=1, \ldots, 7)$ coefficients are given by

$$
C_{3}^{1 v}=\frac{7 \oint \delta\left(g-g_{1}^{*}\right) T_{3}^{1 v}(g)}{\oint \delta\left(g-g_{1}^{*}\right) d g}=7 T_{3}^{1 v}\left(g_{1}^{*}\right)=7 \delta_{7}^{v}
$$

Among $\xi_{3}^{v}$ coefficients, only $\xi_{3}^{7}=e_{3}^{1}$ survives, so the polycrystal piezoelectric modulus (eq. 26) coincides with that of a monocrystal, as it should be in this extreme case.

Cases $\Omega_{0}=5^{\circ}, 10^{\circ}, 20^{\circ}, 30^{\circ}$. Evaluation of (31) by numerical methods, leads to the following results: $C_{3}^{1 v} \approx 0(v=1, \ldots, 6)$, for all the considered $\Omega_{0}$. $C_{3}^{17}$ gives place to the values shown in Table 1 . The synthesis of $\langle d\rangle$ leads to the results drawn in Figure 3 (monocrystal case, $\Omega_{0}=0$, is included).

Table 1 Expansion coefficients for model textures

\begin{tabular}{lccccc}
\hline$\Omega_{0}$ & $0^{\circ}$ & $5^{\circ}$ & $10^{\circ}$ & $20^{\circ}$ & $30^{\circ}$ \\
\hline$C_{3}^{17}$ & 7.000 & 6.828 & 6.256 & 4.765 & 3.058 \\
\hline
\end{tabular}

\section{TEXTURE AND FERROELECTRICITY}

Ferroelectric materials are a subset of piezoelectric ones. In this section we give an ODF approach to the characterization of a ferroelectric polarization process. We also apply the preceding sections' algorithms to the prediction of piezoelectric properties in ferroelectric polycrystals.

Let us consider, as a typical case, a $\mathrm{BaTiO}_{3}$ polycrystal at a temperature above its Curie point, $\mathrm{T}>\mathrm{T}_{\mathrm{c}}$. Sample (perovskite) crystallites belong to cubic, centrosymmetric point group $\mathrm{m} 3 \mathrm{~m}$. At the mentioned temperature, our material is not piezoelectric. As a result of its past history, the sample exhibits a given texture. We describe this texture by the corresponding ODF, CDPF and CIPF, defined accoding to (15), (22) and (23). For the present discussion, we denote by $f^{D}(g), Q_{h}^{D}(y), R_{y}^{D}(h)$ these crystallographic texture functions. They represent the linear-dielectric or "paraelectric" state. Samplecrystal symmetry is characterized by the condition (19), writing $g_{B_{j}}^{D}\left(j=1, \ldots, N_{B}^{D}\right)$ to denote the subgroup of proper rotations contained in the point group associated to the paraelectric crystal symmetry.

Let now temperature decrease under $T_{c}$. The external electric field acting over the sample is null, or weak (far from a saturation field). The paraelectric - ferroelectric phase transition takes place. In the $\mathrm{BaTiO}_{3}$ case, the crystallographic structure loses its centro-symmetric character and passes from cubic to tetragonal. The new point group is $4 \mathrm{~mm}$, a subgroup of $\mathrm{m} 3 \mathrm{~m}$. Cell electric dipole density transforms from a null vector to vector $P_{0}$, pointing along the now longest elementary cell edge. The positive direction of the crystallographic tetragonal axis $Z_{B}^{F}$ is taken, in each domain, along $P_{0}$. A representative crystallite will divide itself into electrically polarized domains. Every domain becomes a crystallite, that follows definite orientation relationships with the other domains that emerged from the same paraelectric crystallite. In barium titanate there appear the so-called $180^{\circ}$ boundaries and, to a lesser extent, those of $90^{\circ}$. Figure 


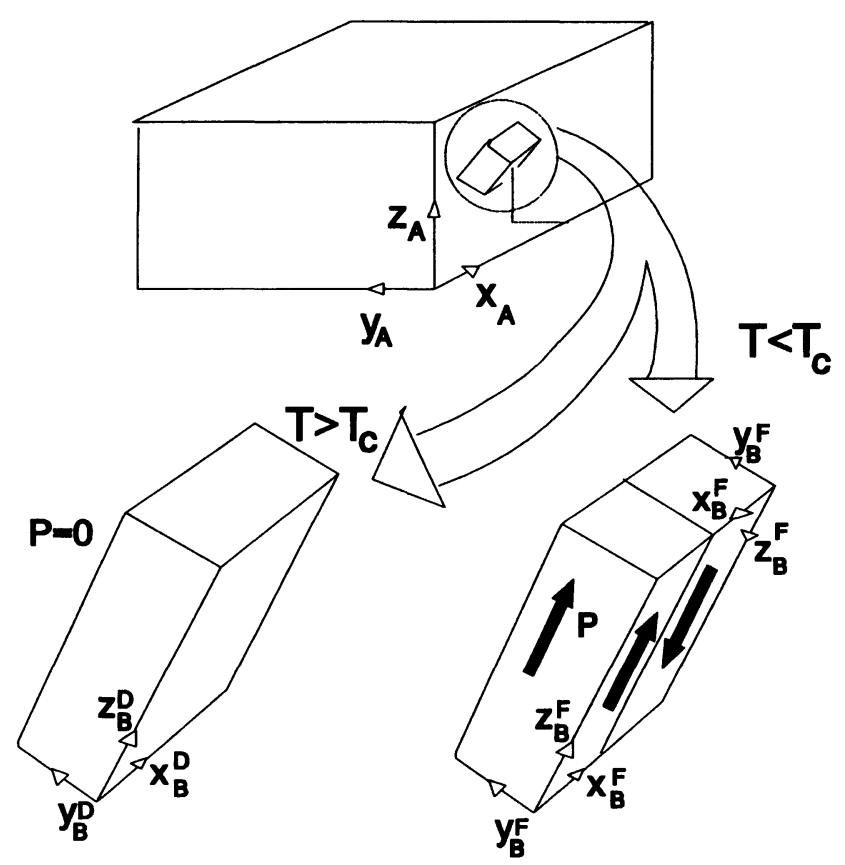

Figure 4 Coordinate systems in the paraelectric. - ferroelectric transition.

4 shows the coordinates that are needed to characterize the situation. $K_{A}\left(x_{A}, y_{A}, z_{A}\right)$ is the "sample reference system". $K_{B}^{D}\left(x_{B}^{D}, y_{B}^{D}, z_{B}^{D}\right)$ is associated to a typical cubic crystallite of the paraelectric condition. In ferroelectric state, several $K_{B}^{F}\left(x_{B}^{F}, y_{B}^{F}, z_{B}^{F}\right)$ systems are required to represent the orientations of the different domains that were born from the analyzed crystallite. The orientation of $K_{B}^{F}$ is given by:

$$
K_{B}^{F}=g \cdot K_{A}=g_{B_{j}}^{D} \cdot K_{B}^{D}
$$

The statistical orientation distribution in the ferroelectric case is described by $f^{F}(g)$, $Q_{h}^{F}(y)$ and $R_{y}^{F}(h)$.

The symmetry of $f^{F}(g)$ is characterized by (19), with group $g_{B_{j}}^{F}\left(j=1, \ldots, N_{B}^{F}\right)$ representing crystal symmetry. The definition domain of $f^{F}(g)$ in Euler space $\left(\Re_{3}^{F}\right)$ is larger than that of $f^{D}(g)\left(\Re_{3}^{D}\right)$ by a factor $N_{B}^{D} / N_{B}^{F}$.

In the paraelectric-ferroelectric transformation a given point $g$ of $\Re_{3}^{D}$ splits into $N_{B}^{D} N_{B}^{F}$ points $g_{B_{j}}^{D}$ g of $\Re_{3}^{F}$. The average value of $f^{F}(g)$ over the last-mentioned points equals $f^{D}(g)$ at the initial $g$ :

$$
f^{D}(g)=\left\langle f^{F}\left(g_{B_{j}}^{D} \cdot \mathrm{g}\right)\right\rangle=\frac{\mathrm{N}_{\mathrm{B}}^{\mathrm{F}}}{\mathrm{N}_{\mathrm{B}}^{\mathrm{D}} \mathrm{j}} \sum^{F}\left(g_{B_{j}}^{D} \cdot g\right)
$$


Electric direct $Q^{P}(y)$ and inverse $R^{P}(h)$ pole figures (EDPF, EIPF) are important from the point of view of electrical properties analysis. These magnitudes are defined by the distribution of polarization directions among the domains in the polycrystal.

$$
\begin{aligned}
& Q^{P}(y)=\frac{d V(y \| P) / V}{d y} \\
& R^{P}(y)=\frac{d V(h \| P) / V}{d h}
\end{aligned}
$$

$P$ denotes the polarization density vector.

Under null or moderate electric fields, the polarization direction in every domain coincides with its $z_{B}^{F}$ axis. The ODF shows both crystallographic and electric characters. The EDPF coincides with the (001) CDPF. The module of the polarization vector is constant over all domains in the sample.

If the applied field $\left(E \| z_{A}\right)$ becomes intense, domain polarization departs from $z_{B}^{F}$ axis, so the ODF loses its electric information. Electric (direct and inverse) pole figures keep their validity.

For very intense electric fields, under so-called saturation conditions, polarization directions coincides with $z_{A}$ for all domains and this implies that the EIPF becomes equal to the $z_{A}$ CIPF.

A sample that was first driven to saturation and then abandoned to $E=0$ evolves to the sate of remanence. In this situation, in an ideal case, equation (36) adopts the form:

$$
f^{F}(g)= \begin{cases}\frac{N_{B}^{D}}{N_{B}^{F}} \cdot \mathrm{f}_{\mathrm{D}} & g \in \Re_{3}^{D} \\ 0 & g \notin \Re_{3}^{D}\end{cases}
$$

To predict in first approximation the piezo-modulus of a $\mathrm{BaTiO}_{3}$ or PZT ceramic that has been drawn to remanent state, one should use equations (26) and (27), with $f^{F}(g)$ satisfying (39).

Under low-field conditions (including remanence) the electric polarization along the $z_{A}$ direction is given by:

$$
\begin{aligned}
& P\left(z_{A}\right)=P_{0} \int Q_{z_{B}}^{F}(y) \cos \left[y, z_{A}\right] d y \\
& P\left(z_{A}\right)=P_{0} \int R_{z_{A}}^{F}(h) \cos \left[h, z_{B}^{F}\right] d h
\end{aligned}
$$

\section{FIBRE TEXTURES IN $\mathrm{BaTiO}_{3}$}

As a concluding application, we model a family of cases with practical importance: the polarization of a $\mathrm{BaTiO}_{3}$ ceramic whose crystallites form a fibre texture. As a result of axial symmetry, the CIPF plays the ODF role. Table 2 schematically shows the microstructure and the CIPF that correspond to different moments in sample's history. Quantitative relations among relevant structural and electrical magnitudes are given.

In (a) the situation for $\mathrm{T}>\mathrm{T}_{\mathrm{c}}$ is described. Our model paraelectric CIPF $\left(R_{z_{A}}^{D}(h)\right)$ 


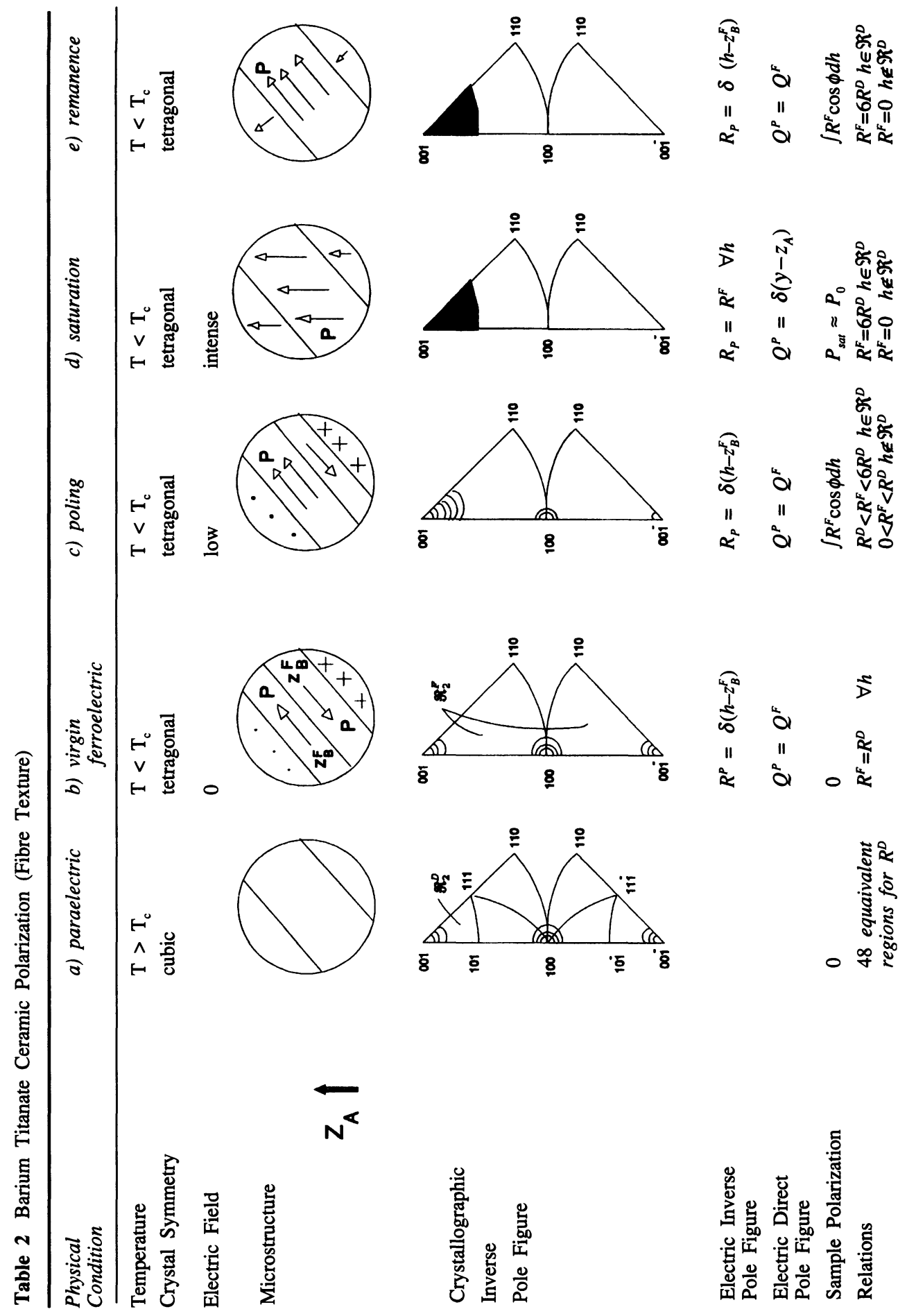


consists of a component, say a gaussian-shaped one, centrered around the [001] direction. By crystal symmetry, this component reproduces itself around all nodes of the form $<001>$ in the projection sphere. The irreducible region of this CIPF, $\Re_{2}^{D}$, is the triangle $<(001)$ (101) (111) $>$ of the Figure. A larger domain has been drawn for comparison with the following cases. Densities $R_{z_{A}}^{D}(h)$ in the neighborhood of (100) and (010) (for example) are equal to those associated with corresponding points near (001). Under the considered conditions the sample is paraelectric, it makes no sense to define $R^{P}(h)$.

In (b) the sample has been cooled to $\mathrm{T}<\mathrm{T}$, without external field. Crystallites break up into polar domains. Crystal structure and symmetry transform. Every domain shows now electric dipole. Texture preserves its axial symmetry. Paraelectric CIPF gives its place to ferroelectric $\left(R_{z_{A}}^{F}(h)\right.$. The irreducible domain for $R_{z_{A}}^{F}(h)\left(\Re_{2}^{F}\right)$ becomes $N_{B}^{D} / N_{B}^{F}=48 / 8=6$ times larger than $\Re_{2}^{D}$.

Cases (c), (d) and (e) represent respectively polarization saturation and remanence.

We apply the relations of case (e) to the determination of remanence and piezoelectric logitudinal modulus in two particular cases: a) a ceramic without initial crystallographic texture, polarized under a strong axially symmetric field. b) a sharp fibre ceramic with a gaussian texture of type (30), broadening $\Omega_{0}=10^{\circ}$.

The considered (final) CIPFs, so normalized that their integrals equal $4 \pi$, are given by:

a) no initial texture; final texture due to polarization.

$$
R_{z_{A}}^{F}(h)= \begin{cases}6 & h \in \Re_{2}^{D} \\ 0 & h \notin \Re_{2}^{D}\end{cases}
$$

b) sharp gaussian texture

$$
R_{z_{A}}^{F}(h)=\left\{\begin{array}{cc}
132 e^{-(18 \phi / \pi)^{2}} & h \in \Re_{2}^{D} \\
0 & h \notin \Re_{2}^{D}
\end{array}\right.
$$

$\phi$ is the polar angle, measured in radians.

For calculation of remanent polarization, equation (41) adopts the form:

$$
P=8 P_{0} \int_{\beta=0}^{\pi / 4} d \beta \int_{0}^{\phi_{1}} R_{z_{A}}^{F}(h) \cos \phi \sin \phi d \phi
$$

To predict piezoelectric longitudinal modulus, we need the coefficients $C_{l}^{\mu}$, with $l=1,3 ; \mu=1$. They are calculated by means of the following integrals:

$$
C_{l}^{1}=8 \int_{\beta=0}^{\pi / 4} d \beta \int_{\phi=0}^{\phi_{1}} R_{z_{A}}^{F}(\phi, \beta) k_{l}^{1}(\phi, \beta) \sin \phi d \phi
$$




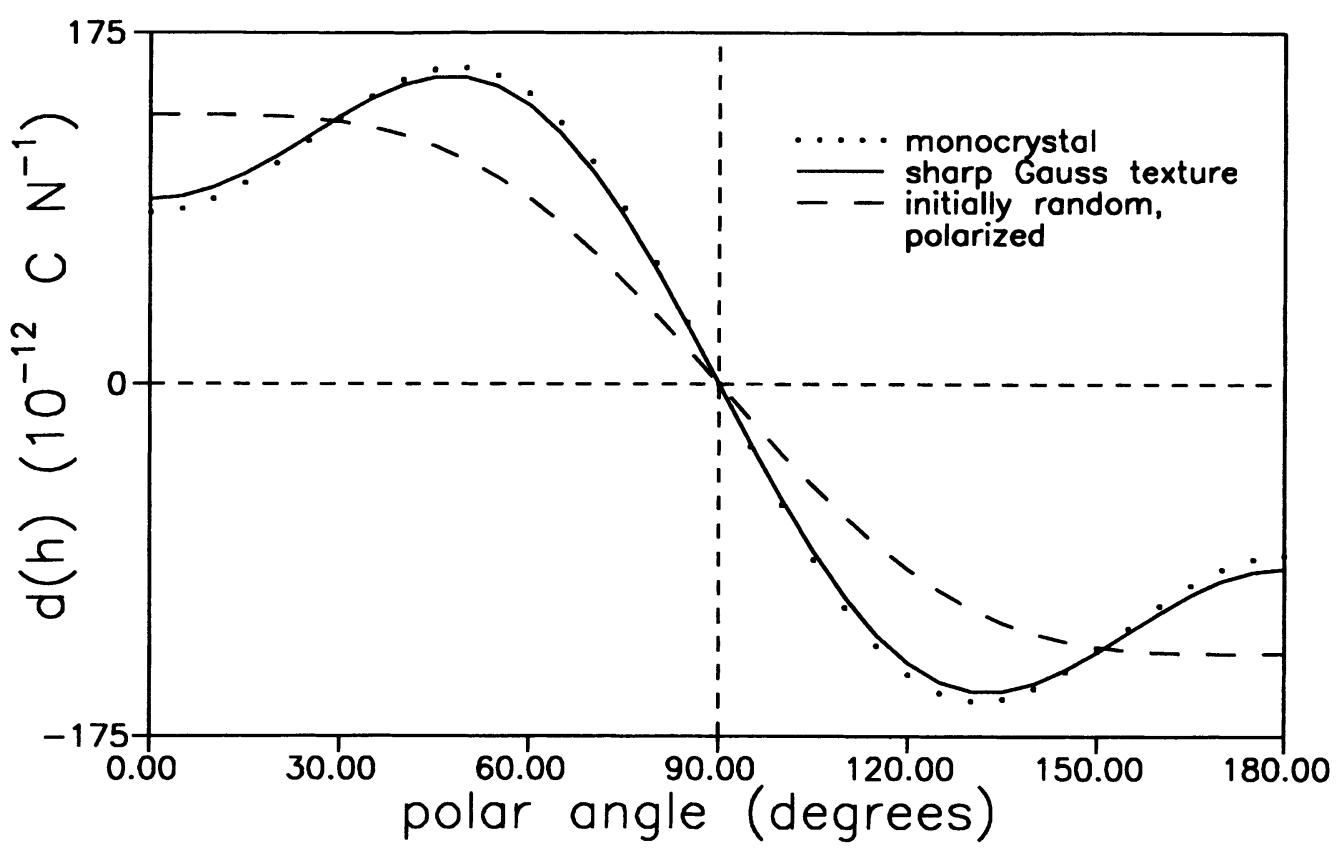

Figure $5 \mathrm{BaTiO}_{3}$ Ceramics. Piezoelectricity.

with tetragonal spherical harmonics as in equation (12). In (44) and (45) the integral upper limit in $\phi$ is given by the condition:

$$
\cot \phi_{1}=\cos \beta
$$

Numerical calculations lead to the following results for remanent polarization:

$$
\begin{gathered}
P=0.83 P_{0} \quad \text { (polarization texture) } \\
P=0.985 P_{0} \quad\left(\text { gaussian texture, } \Omega_{0}=10^{\circ}\right)
\end{gathered}
$$

Longitudinal piezoelectric moduli are found to follow the curves plotted in Figure 5. One interesting result is obtained for $\phi=0$ : the highest piezoelectric modulus corresponds to the initially random polycrystalline sample. This finding characterizes the experimental tendency, as may be seen by comparing the monocrystal and polycrystal $d_{33}$ values reported in the cited literature. 
APPENDIX I: TRICLINIC BIDIMENSIONAL SPHERICAL HARMONICS

$$
\begin{gathered}
K_{l}^{\mu}(\phi, \beta) \quad(l=1,3) \\
K_{1}^{1}=\frac{1}{2} \sqrt{\frac{3}{\pi}} \sin \phi \sin \beta \\
K_{1}^{2}=\frac{1}{2} \sqrt{\frac{3}{\pi}} \cos \phi=\frac{1}{\sqrt{2 \pi}} P_{1}(\phi) \\
K_{1}^{3}=\frac{1}{2} \sqrt{\frac{3}{\pi}} \sin \phi \cos \beta \\
K_{3}^{1}=\frac{1}{8} \sqrt{\frac{70}{\pi}} \sin { }^{3} \phi \sin 3 \beta \\
K_{3}^{2}=\frac{1}{4} \sqrt{\frac{105}{\pi}}\left(\cos \phi-\cos ^{3} \phi\right) \sin 2 \beta \\
K_{3}^{6}=\frac{1}{4} \sqrt{\frac{105}{\pi}}\left(\cos \phi-\cos ^{3} \phi\right) \cos 2 \beta \\
K_{3}^{5}=\frac{1}{8} \sqrt{\frac{42}{\pi}}\left(4 \sin \phi-5 \sin ^{3} \phi\right) \cos \beta \\
K_{3}^{4}=\frac{1}{4} \sqrt{\frac{7}{\pi}}\left(5 \cos ^{3} \phi-3 \cos ^{3} \phi\right)=\frac{1}{\sqrt{2 \pi}} P_{3}(\phi) \\
\left.\sin { }^{3} \phi \cos 3 \beta\right) \sin \beta
\end{gathered}
$$




\section{APPENDIX II: TRIDIMENSIONAL SYMMETRY-ADAPTED SPHERICAL HARMONICS. TRICLINIC-TRIGONAL CASE.}

$$
\begin{gathered}
T_{3}^{11}=\frac{1}{8}\left[(1+\cos \phi)^{3} \sin \left(3 \varphi_{2}+3 \varphi_{1}\right)-(1-\cos \phi)^{3} \sin \left(3 \varphi_{2}-3 \varphi_{1}\right)\right] \\
T_{3}^{12}=\frac{\sqrt{6}}{8}\left[-(1+\cos \phi)^{2} \sin \phi \cos \left(3 \varphi_{2}+2 \varphi_{1}\right)+(1+\cos \phi)^{2} \sin \phi \cos \left(3 \varphi_{2}-2 \varphi_{1}\right)\right. \\
T_{3}^{13}=\frac{\sqrt{15}}{8}\left[-(1+\cos \phi) \sin ^{2} \phi \sin \left(3 \varphi_{2}+\varphi_{1}\right)+(1-\cos \phi) \sin ^{2} \phi \sin \left(3 \varphi_{2}-\varphi_{1}\right)\right] \\
T_{3}^{14}=\frac{\sqrt{10}}{4} \sin ^{3} \phi \cos 3\left(\varphi_{2}-\frac{\pi}{2}\right) \\
T_{3}^{15}=\frac{\sqrt{15}}{8}\left[-(1+\cos \phi) \sin ^{2} \phi \cos \left(3 \varphi_{2}+\varphi_{1}\right)+(1-\cos \phi) \sin ^{2} \phi \cos \left(3 \varphi_{2}-\varphi_{1}\right)\right] \\
T_{3}^{16}=\frac{\sqrt{6}}{8}\left[(1+\cos \phi)^{2} \sin ^{2} \sin \left(3 \varphi_{2}+2 \varphi_{1}\right)+(1+\cos \phi)^{2} \sin \phi \sin \left(3 \varphi_{2}-2 \varphi_{1}\right)\right] \\
T_{3}^{17}=\frac{1}{8}\left[(1+\cos \phi)^{3} \cos \left(3 \varphi_{2}+3 \varphi_{1}\right)-(1-\cos \phi)^{3} \cos \left(3 \varphi_{2}-3 \varphi_{1}\right)\right]
\end{gathered}
$$

\section{References}

Berlincourt, D. A., Curran, D. R. and Jaffe, H. (1964). Piezoelectric and Piezomagnetic Materials and Their Function in Transducers. Physical Acoustics, Volume 1 Part A, Ed. by Mason, W. P. Academic Press, New York.

Bunge, H. J. (1982). Texture Analysis in Materials Science. Butterworths Publ., London.

Bunge, H. J. (1989). Texture and Magnetic Properties. Textures and Microstructures 11, 75-91.

Gallego-Juárez, J. A. (1989). Piezoelectric Ceramics and Ultrasonic Transducers. J. Phys. E: Sci. Instrum. 22, 804-816.

Ghomshei, M. M., Narod, B. B., Templeton, T. L., Arrott, A. S. and Russell, R. D. (1988) Piezoelectric pole figure of a vein quartz sample Textures and Microstructures 7, 303-316.

Helming, K. and Matthies, S. (1984). On the Interpretation of Orientation Distributions and Qualitative Ghost Corrections for Hexagonal-Orthorhombic Textures. Phys. Stat. Sol. (b) 126, 43-52.

IEEE Standards on Piezoelectricity (1987). ANSI/IEEE Std. 176.

Ikeda, T. (1990). Fundamentals of Piezoelectricity. Oxford Univ. Press, Oxford.

International Tables of Crystallography (1983). Reidel Publ. Co., Dordrech.

Mitsui, T., Nakamura, E. (1989). Ferroelectric and Related Substances. Subvolume a: Oxides. Numerical Data dan Functional Relationships in Science and Technology. Landolt-Börnstein New Series, Group III, Vol. 28. Springer-Verlag, Berlin.

Nye, J. F. (1957). The Physical Properties of Crystals. Clarendon Press, Oxford.

Raymond, O., Fuentes, L. and Gómez, J. (1995). Computer-Oriented Real Spherical Harmonics for Texture and Properties Analysis (to be published).

Zgonik, M., Bernasconi, P. Duelli, M. et al. (1994). Dielectric, Elastic, Piezoelectric, Electro-optic, and Elasto-optic Tensors of $\mathrm{BaTiO}_{3}$ Crystals. Physical Review B, 50, 5941-5949. 